Original paper

\title{
Diagnosis of spontaneous bacterial peritonitis in children using leukocyte esterase reagent strips and granulocyte elastase immunoassay
}

\author{
Alif Abd El-Hakim Allam¹, Sara Mohammed Eltaras ${ }^{1}$, Mohsen Hassan Hussin', El-Sayed Ibrahem Salama', \\ Olfat Mohamed Hendy², Maha Moawad Allam², Haidy Mohammed Zakaria ${ }^{3}$ \\ 'Department of Pediatric Hepatology, Gastroentrology and Nutrition, National Liver Institute, Menoufia University, 32511 Shebin El-koom, \\ Menoufia, Egypt \\ ${ }^{2}$ Clinical Pathology Department, National Liver Institute, Menoufia University, 32511 Shebin El-koom, Menoufia, Egypt \\ ${ }^{3}$ Pediatric Department, Quesna Central Hospital, Ministry of Health, Menoufia, Egypt
}

\begin{abstract}
Aim of the study: We aimed to assess the utility and rapidity of granulocyte elastase (GE) latex immunoassay and leukocyte esterase (LE) reagent strips for the diagnosis of spontaneous bacterial peritonitis (SBP) in hepatic children with ascites.

Material and methods: This study included 80 ascitic fluid (AF) samples from 45 patients with chronic liver diseases. They were divided into 2 groups (SBP and non-SBP groups). White blood cells $>500$ cell/ $/ \mathrm{mm}^{3}$ or polymorphonuclear leukocytes $>250$ cell/ $/ \mathrm{mm}^{3}$ were considered as a positive result for the diagnosis of SBP. The AF obtained at bedside was immediately tested with an LE enzyme reagent strip, and another sample was aliquoted for measurement of serum GE.

Results: On doing LE dipstick strips, all the non-SBP group gave no coloration with LE strips while $62 \%$ of the SBP group gave coloration. LE strips had accuracy of $86.25 \%$ in differentiating SBP and non-SBP at a cut-off value of 1 (color grade 1). The diagnostic performance of GE in differentiating SBP and non-SBP was assessed and showed accuracy of $70 \%$ for a cutoff value of $123.5 \mathrm{ng} / \mathrm{ml}$.

Conclusions: Application of LE reagent strips is a bedside, rapid, easy-to-use, and inexpensive method for diagnosis of SBP. It has an accuracy of $86.25 \%$ in differentiating SBP and non-SBP, which is higher than more complicated and delayed methods such as GE latex immunoassay, which has an accuracy of $70 \%$.
\end{abstract}

Key words: spontaneous bacterial peritonitis, granulocyte elastase, leukocyte esterase.

Address for correspondence

Haidy Mohammed Zakaria, MD, Pediatric Department, Quesna Central Hospital, Ministry of Health, Menoufia, Egypt, phone: +20 1005768306, e-mail: drhaydi2000@gmail.com

\section{Introduction}

Spontaneous bacterial peritonitis (SBP) arises almost exclusively in patients with cirrhosis. It has been described as an ascitic fluid (AF) infection in the absence of any intra-abdominal, surgically treatable source of infection. It is diagnosed when there is an elevated AF absolute polymorphonuclear leukocyte (PMN) count with or without positive AF culture (i.e. $\geq 250$ cells $\left./ \mathrm{mm}^{3}\right)$ [1].
The count of AF PMNs is operator-dependent and lysis of PMN cells during transport to the laboratory may lead to false-negative results. Furthermore, AF culture is insensitive and leads to delay in diagnosis by several days. A delay in antibiotic therapy entails a high mortality rate; therefore, considerable efforts have been invested in developing a rapid and reliable test for the diagnosis of SBP [2].

Using bedside, rapid, easy-to-use, and inexpensive methods for diagnosis of SBP will help in early diagnosis and appropriate intervention [3]. 
The aim of this study is to assess the utility of granulocyte elastase (GE) immunoassay and rapidity of leucocyte esterase (LE) reagent strips for the diagnosis of SBP in cirrhotic children with ascites.

\section{Material and methods}

This case control study included 80 AF samples from 45 patients with chronic liver diseases. They were divided into 2 groups (SBP and non-SBP groups). The number of paracentesis procedures per patient ranged from one to four, with an average of $2 \mathrm{AF}$ samples/patient. The SBP group included $40 \mathrm{AF}$ samples with $\mathrm{PMN}$ count in $\mathrm{AF} \geq 250$ cells per $\mathrm{mm}^{3}$ with or without culture positive or positive AF culture with $\mathrm{PMN}<250$ per $\mathrm{mm}^{3}$. Non-SBP group: included $40 \mathrm{AF}$ samples with $\mathrm{PMN}$ count in $\mathrm{AF}<250$ cells per $\mathrm{mm}^{3}$ with AF culture negative.

All patients in this study were subjected to full history taking, thorough clinical examination, and routine investigations. AF samples were carried out under strict sterile conditions with the patient in a supine position, ultrasound guided or during tapping of a large amount of ascites.

AF samples were investigated for AF culture and sensitivity, and total leucocytic count (TLC) with its differential. Biochemical AF tests, such as triglycerides, cholesterol, bilirubin, albumin, total protein, glucose, amylase, lipase and lactic dehydrogenase, were performed.

After the paracentesis, the AF specimen was collected in a clean sterile container. Then the LE test strip

Table 1. Etiological diagnosis of the ascitic patients

\begin{tabular}{lcc}
\hline \multirow{2}{*}{ Etiological diagnosis } & \multicolumn{2}{c}{ Studied group $\boldsymbol{N}=\mathbf{4 5}$} \\
\cline { 2 - 3 } & $\boldsymbol{n}$ & $\%$ \\
\hline Biliary atresia & 10 & $22.2 \%$ \\
Budd-Chiari syndrome & 9 & $20.0 \%$ \\
Autoimmune hepatitis & 5 & $11.1 \%$ \\
Progressive familial intrahepatic cholestasis & 5 & $11.1 \%$ \\
Congenital hepatic fibrosis & 3 & $6.7 \%$ \\
Tyrosinemia & 2 & $4.4 \%$ \\
Wilson disease & 2 & $4.4 \%$ \\
Glycogen storage disease & 1 & $2.2 \%$ \\
Sclerosing cholangitis & 1 & $2.2 \%$ \\
Alagille syndrome & 1 & $2.2 \%$ \\
Caroli syndrome & 1 & $2.2 \%$ \\
Galactosemia & 1 & $2.2 \%$ \\
Hepatitis C virus & 1 & $2.2 \%$ \\
Giant cell hepatitis with AlHA & 1 & $2.2 \%$ \\
Portal vein thrombosis & 1 & $2.2 \%$ \\
Undiagnosed liver failure & 1 & $2.2 \%$ \\
\hline
\end{tabular}

(Combur test UX; Roche Diagnostics GmbH, D-68298 Mannheim, Germany) was dipped for no longer than 1 second. After 60 seconds the reaction color in the test area was compared against the color scale (4 grade scale from 0 to 3 ) on the label.

Human GE was measured by ELISA technique and supplied by BIOSCIENCE, USA, with catalog number BMS269, Affymetrix.

\section{Methods of data analysis and statistical evaluation}

Data were collected and entered to the computer using SPSS (Statistical Package for the Social Science) program for statistical analysis version 21 (Armonk, NY: IBM Corp.). Quantitative data will be shown as mean \pm SD. Qualitative data will be expressed as frequency and percent. The chi-square test $\left(\chi^{2}\right)$ was used to evaluate the association between qualitative variables. The Mann-Whitney test $(z)$ and independent sample $t$ test were used to evaluate the association between 2 sets of quantitative data. The results of comparing the correlation between two continuous variables are indicated by the correlation coefficient $(r)$ using correlation analysis. The clinical performance of GE and LE in differentiating SBP from non-SBP were assessed by the receiver operating characteristic (ROC) curves. Sensitivity, specificity, positive predictive value (PPV) and negative predictive value (NPV) were presented as percentages. The $P$ (probability) value is considered to indicate statistically significance if it is less than 0.05 .

\section{Results}

Forty-five patients with ascites (64.4\% males, $35.6 \%$ females, mean age 4.5 years) with different etiologies were included in the study (Table 1).

Analysis of the results showed that fever, abdominal pain and abdominal tenderness were the only significant clinical presentations in patients with SBP compared to the non-SBP group $(<0.0001)$. Seventeen $(68 \%)$ cases of the SBP group had fever on presentation without a clinically apparent source of fever, while only 2 cases of non-SBP had fever (one case had pharyngitis and the other had otitis media). Abdominal pain and abdominal tenderness were detected in $92 \%$ and $88 \%$ respectively in the SBP group. Regarding the laboratory parameters, there was no difference regarding either complete blood count (CBC) parameters or liver function tests.

On analysis of ascitic samples, TLC and neutrophils were found to be higher in the SBP group while lymphocytes were higher in the non-SBP group. Sixty per- 
cent of TLC of the SBP group were neutrophils while 97.5\% of TLC of the non-SBP group were lymphocytes (Table 2). As regards culture of the ascitic sample, 20\% of SBP were culture positive (5 cases had E. coli and 3 had Klebsiella) while none of the other group were positive.

On doing the GE immunoassay, GE was significantly higher in the SBP $(3073.6 \pm 7082.78)$ than the non-SBP group $(430 \pm 1029.1)$ ( $p$ value $<0.05)$. The diagnostic performance of GE in differentiating SBP and non-SBP was assessed and showed sensitivity, specificity, PPV, NPV and accuracy of $70 \%$ for each at a cut-off value of $123.5 \mathrm{ng} / \mathrm{ml}$. Granulocyte elastase was positively correlated with TLC and neutrophil percent in the ascitic sample and showed a negative correlation with the lymphocytic percent in the ascitic sample $(p<0.05)$.

On doing LE dipstick strips, all the non-SBP group gave no coloration with LE strips while $62 \%$ of the SBP group gave coloration. $17.5 \%$ of SBP gave positive + , $22.5 \%$ gave positive ++ and $22.5 \%$ gave positive +++ .

Leukocyte esterase strips in differentiating SBP and non-SBP had sensitivity of $62.5 \%$, specificity of $100 \%$, PPV of $100 \%$, NPV of $72.5 \%$ and accuracy of $86.25 \%$ at cut-off value of 1 (color grade 1). Using the color grade of LE strips in predicting TLC count in ascitic sample, no coloration indicates TLC count $<750$ cell/ $\mathrm{ml}$ with accuracy of $86.75 \%$. Color grade + indicates TLC $>750 \mathrm{cell} / \mathrm{ml}$ with accuracy $86.75 \%$. Color grade ++ indicates TLC $>950 \mathrm{cell} / \mathrm{ml}$ with accuracy $83.35 \%$. Color grade +++ indicates TLC $>1950 \mathrm{cell} / \mathrm{ml}$ with accuracy $75.5 \%$ (Fig. 1).

By use of the color grade of LE strips in predicting PMN cell count in ascitic sample, we found that: no coloration detects $\mathrm{PMN}$ count $<310$ cells $/ \mathrm{ml}$ with accuracy of $85.05 \%$; color grade + detects PMN $>310$ cells/ $\mathrm{ml}$ with accuracy $85.05 \%$; color grade ++ detects PMN $>691$ cells $/ \mathrm{ml}$ with accuracy $89.15 \%$; color grade +++ detects PMN > 1577 cells $/ \mathrm{ml}$ with accuracy $82.15 \%$ (Fig. 2).

\section{Discussion}

SBP is life-threatening in decompensated liver disease, which needs close monitoring, early diagnosis and rapid intervention. Bacterial translocation is considered the key step in SBP pathogenesis; it is the "passage" of bacteria from the lumen to the mesenteric lymph nodes and thereafter to the blood stream and other extra-intestinal sites. It represents failure of a group of defensive factors to contain bacteria within the bowel. Bacterial overgrowth in association with impairment of the intestinal barrier (probably a consequence of vascular
Table 2. Ascitic fluid TLC and its differential in the SBP and non-SBP groups

\begin{tabular}{lccc}
\hline & $\begin{array}{c}\text { SBP } \\
N=40\end{array}$ & $\begin{array}{c}\text { Non-SBP } \\
N=40\end{array}$ & p-value \\
\hline TLC (cells/mm $)$ & $3432 \pm 4738$ & $345 \pm 95.9$ & $<0.0001$ \\
\hline Neutrophil (\%) & $57.33 \pm 29.26$ & $27.7 \pm 11.51$ & $<0.0001$ \\
\hline PMN cell count (cells/mm $\left./ \mathrm{mm}^{3}\right)$ & $2558 \pm 4329$ & $95.52 \pm 44.93$ & $<0.0001$ \\
\hline Lymphocyte (\%) & $41.73 \pm 29.80$ & $71.98 \pm 11.53$ & $<0.0001$ \\
\hline $\begin{array}{l}\text { TLC differential } \\
\text { mainly neutrophils } \\
\text { mainly lymphocytes }\end{array}$ & $24(60.0 \%)$ & $1(2.5 \%)$ & $<0.0001$ \\
\hline Positive AF culture results & $8(20.0 \%)$ & $0(0.0 \%)$ & 0.005 \\
\hline
\end{tabular}

stasis due to portal hypertension), alterations of local immune defenses, slow motility of the bowel in patients with cirrhosis and reduced opsonic activity precede the episodes of bacterial translocation. More recently detection of translocation of bacterial products through the intestinal wall has been associated with production of many cytokines which cause over-activation of the sepsis syndrome pathways [4].

Fever, abdominal pain and abdominal tenderness were the only significant clinical presentations in patients with SBP compared to the non-SBP group. In agreement with our results, Bibi et al. (2015) found that fever was higher in SBP than non-SBP [5]. Abdominal pain and abdominal tenderness were detected in $92 \%$ and $88 \%$ respectively in the SBP group. These results were higher than those reported by Wallersted et al. (2007), who stated that abdominal pain and tenderness were detected in $70 \%$ of SBP cases [6]. Regarding the laboratory parameters, there was no difference regarding either $\mathrm{CBC}$ parameters or liver function tests.

TLC and neutrophils were higher in AF of the SBP group, while lymphocytes were higher in the non-SBP group. Sixty percent of TLC of the SBP group were neutrophils while $97.5 \%$ of TLC of the non-SBP group were lymphocytes. Abulseoud et al. (2016) stated that, although ascitic total WBC count increases in SBP cases, it suffers from low specificity because a large proportion of patients with sterile ascites have increased white blood cell counts [7].

GE was significantly higher in the SBP (3073.6 $\pm 7082.78)$ than the non-SBP group $(430 \pm 1029.1)$. GE at cut-off value of $123.5 \mathrm{ng} / \mathrm{ml}$ had accuracy of $70 \%$ in differentiating SBP from non-SBP samples. Yamazaki et al. (2011) found that the ROC curve showed that ascitic GE by immunoassay enabled discrimination between SBP and non-SBP, and a cut-off value of $49.5 \mathrm{ng} / \mathrm{ml}$ had a sensitivity of $85.7 \%$ and specificity of $97.7 \%$ [8]. GE was positively correlated with TLC 


\section{TLC count in ascitic sample}
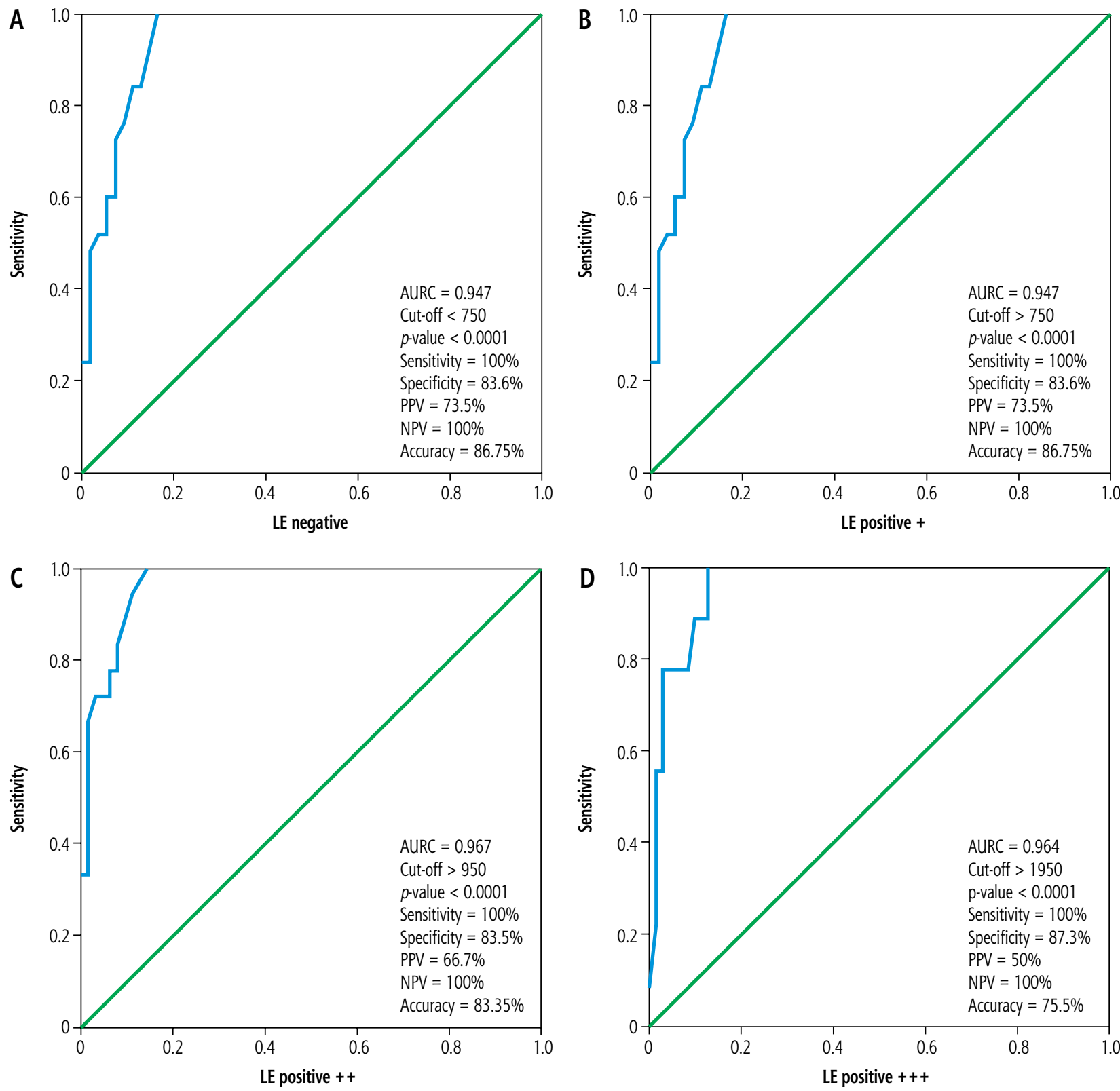

Fig. 1. ROC curve of color grades of LE strips in relation to TLC count in ascitic sample

and neutrophil percent and negatively correlated with the lymphocytic percent in AF $(p<0.05)$.

On doing LE dipstick strips, all non-SBP group samples showed no change in strip color while $62 \%$ of the SBP group showed different grades of discoloration, which led us to assess the predictive power of each color grade in predicting different levels of TLC and PMN cells at bedside.

LE strips at cut-off value of 1 (color grade 1) had an accuracy of $86.25 \%$ in differentiating SBP and nonSBP patients. Comparable results were reported by Koulaouzidis (2011), when using the same strips to diagnose SBP in 136 specimens by using grade 2 as a cut- off scale, and found the sensitivity, specificity, PPV and NPV of $83 \%, 99 \%, 91 \%$ and $98 \%$ respectively [9]. Also, Castellote and Xiol (2008) studied 228 samples of AF and diagnosed 52 episodes of SBP, using different type of strips (Autionsticksâ, A. Menarini Diagnostics, Firenze, Italy). The sensitivity and specificity of the reagent strip were $96 \%$ and $89 \%$, respectively [10].

\section{Conclusions}

Application of leukocyte esterase reagent strips is a bedside, rapid, easy-to-use, and inexpensive method for diagnosis of SBP. It has an accuracy of $86.25 \%$ in 
PMN cell count in ascitic sample
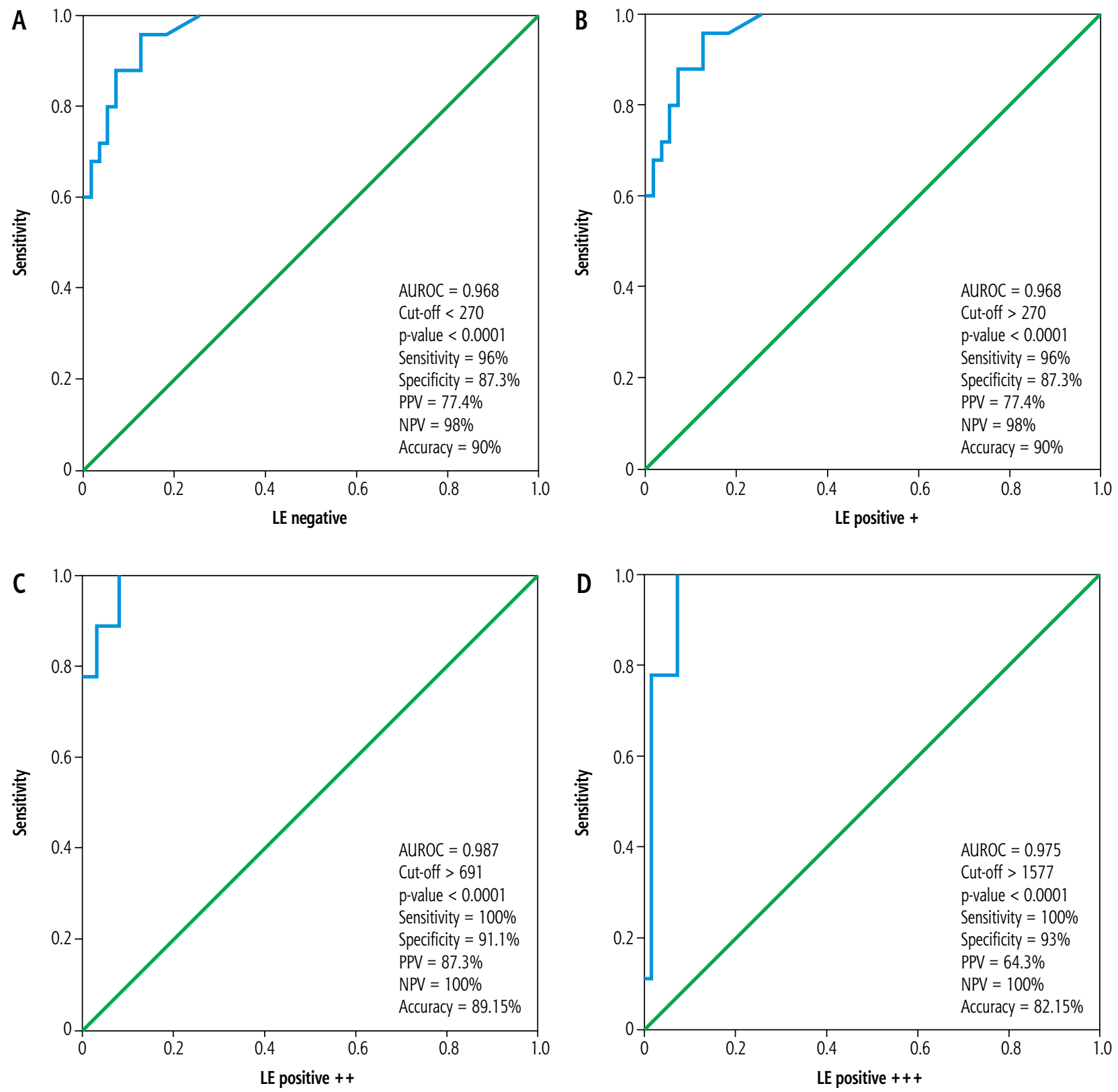

1-Specificity

Fig. 2. ROC curve color grades of LE strips in relation to PMN cell count in ascitic sample

differentiating SBP and non-SBP, which is higher than more complicated methods such as GE latex immunoassay, which has an accuracy of $70 \%$.

\section{Acknowledgements}

We would like to express our gratitude to our colleagues in the Department of Pediatric Hepatology, Gastroenterology and Nutrition, National Liver Institute, Menoufia University, who provided insight and expertise that greatly assisted the research. We would also like to thank all the patients who participated in the study.

\section{Disclosure}

Authors report no conflict of interest.

\section{References}

1. Lata J, Stiburek O, Kopacova M. Spontaneous bacterial peritonitis: a severe complication of liver cirrhosis. World J Gastroenterol 2009; 15: 5505-5510. 
2. Parsi MA, Saadeh SN, Zein NN, et al. Ascitic fluid lactoferrin for diagnosis of spontaneous bacterial peritonitis. Gastroenterology 2008; 135: 803-807.

3. Roberts GH, Ramsey MK. Guidelines for interpretation of the routine urinalysis. Consultant Pharmacist 1990; 5: 748-756.

4. Koulaouzidis A, Bhat S, Karagiannidis A, et al. Spontaneous bacterial peritonitis. Postgrad Med J 2007; 83: 379-383.

5. Bibi S, Ahmed W, Arif A, et al. Clinical, laboratory and bacterial profile of spontaneous bacterial peritonitis in chronic liver disease patients. J Coll Physicians Surg Pak 2015; 25: 95-99.

6. Wallerstedt S, Olsson R, Simren M, et al. Abdominal tenderness in ascites patients indicates spontaneous bacterial peritonitis. Eur J Intern Med 2007; 18: 44-47.

7. Abulseoud A, Ibrahim H, Essa A, et al. Study of the role of ascitic fluid lactoferrin levels in the diagnosis of spontaneous bacterial peritonitis. Menoufia Med J 2016; 29: 464-467.

8. Yamazaki M, Sano R, Kuramoto C, et al. Rapid detection of spontaneous bacterial peritonitis by granulocyte elastase latex immunoassay and reagent strip. Rinsho Byori 2011; 59: 549-558.

9. Koulaouzidis A. Diagnosis of spontaneous bacterial peritonitis: an update on leucocyte esterase reagent strips. World J Gastroenterol 2011; 17: 1091-1094.

10. Castellote J, Xiol X. Reagent strips and spontaneous bacterial peritonitis. Alimentary Pharm Ther 2008; 28: 660; author reply 1. 